\title{
Automated Registration of Whole-Body Follow-Up MicroCT Data of Mice
}

\author{
Martin Baiker ${ }^{1}$, Marius Staring ${ }^{1}$, Clemens W.G.M. Löwik ${ }^{2}$, \\ Johan H.C. Reiber ${ }^{1}$, and Boudewijn P.F. Lelieveldt ${ }^{1,3}$ \\ 1 Div. of Image Processing, Leiden University Medical Center, The Netherlands \\ b.p.f.lelieveldt@lumc.nl \\ http://www.lkeb.nl \\ 2 Dept. of Endocrinology, Leiden University Medical Center, The Netherlands \\ 3 ICT group, Dept. of Mediamatics, Delft University of Technology, The Netherlands
}

\begin{abstract}
In vivo MicroCT imaging of disease models at multiple time points is of great importance for preclinical oncological research, to monitor disease progression. However, the great postural variability between animals in the imaging device complicates data comparison.

In this paper we propose a method for automated registration of whole-body MicroCT follow-up datasets of mice. First, we register the skeleton, the lungs and the skin of an articulated animal atlas (Segars et al. 2004) to MicroCT datasets, yielding point correspondence of these structures over all time points. This correspondence is then used to regularize an intensity-based B-spline registration. This two step approach combines the robustness of model-based registration with the high accuracy of intensity-based registration.

We demonstrate our approach using challenging whole-body in vivo follow-up MicroCT data and obtain subvoxel accuracy for the skeleton and the skin, based on the Euclidean surface distance. The method is computationally efficient and enables high resolution whole-body registration in $\approx 17$ minutes with unoptimized code, mostly executed single-threaded.
\end{abstract}

\section{Background}

The possibility to scan the entire body of small animals with dedicated hardware in vivo offers great benefits for preclinical research, because it allows to follow e.g. pathology development over time within the same subject. This excludes intersubject variability and has ethical and economical benefits.

A problem that arises with imaging entire bodies is the potentially large postural variability of animals that are imaged at different time points (Fig. 1). This significantly complicates data examination, because researchers have to 'align' structures of interest visually and navigate through large whole-body datasets. For some applications, dedicated animal holders can be used to reduce the postural variability. However, such holders may influence the study, e.g. by obstructing light in optical imaging based studies.

To deal with the problem of high postural variability, in [1] we presented a robust method for registration between the skeleton, the lungs and the skin of 

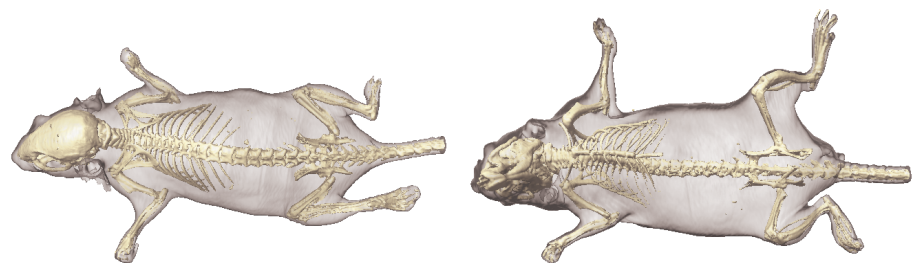

Fig. 1. Demonstration of the large variability in animal posture between different scans. Shown are subjects scanned in prone (left) and supine (right) position.

a mouse atlas (MOBY [2]) and whole-body MicroCT data of mice. We subsequently used the point correspondences on these structures to map the remainder of the body using Thin Plate Spline (TPS) interpolation. However, in areas with few correspondences, the accuracy of the mapping may be limited.

In this paper we aim at improving the accuracy of the TPS mapping by integrating intensity information during the registration. We present an accurate, time efficient and highly robust method for registration of follow-up MicroCT datasets that contain articulated objects. This we achieve by regularizing an intensity-based registration criterion with the Euclidean distance metric, based on pointsets of anatomical correspondences. We evaluate the method using noncontrast-enhanced MicroCT data of eight animals, imaged at two time points.

\section{Previous Work}

Several strategies are described in the literature that focus on registration of images with multiple structures of interest with varying structural properties. Staring et al. 3] describe an approach that adds a local rigidity penalty term to the registration function to penalize rigid object deformations. Somayajula et al. 4] present an intensity-based registration of whole-body MicroCT follow-up datasets of mice using a scale-space approach. A method that relies on skeleton segmentations from MicroCT is described in Li et al. [5]. The skeletons are aligned using nonrigid robust point matching, followed by intensity-based nonrigid registration based on radial basis functions. Suh et al. [6] register the skeleton using extended demons with subsequent intensity-based registration using normal demons. These approaches exploit the high CT contrast to avoid unrealistic bone deformation without [4] and with 356] using the skeleton explicitly. All methods may suffer from local minima when bones are in close proximity, but especially in case of large postural variability.

A possibility to increase the robustness of whole-body registration is to model and register individual parts of an animal. Approaches range from registration of individual Volumes Of Interest and subsequent interpolation (block-matching), that do not take relationships between VOIs into account, to methods that register structures of interest simultaneously or hierarchically. Articulated registration methods are based on realistic modeling of joints and were applied for example to mouse hind limbs [7]. 


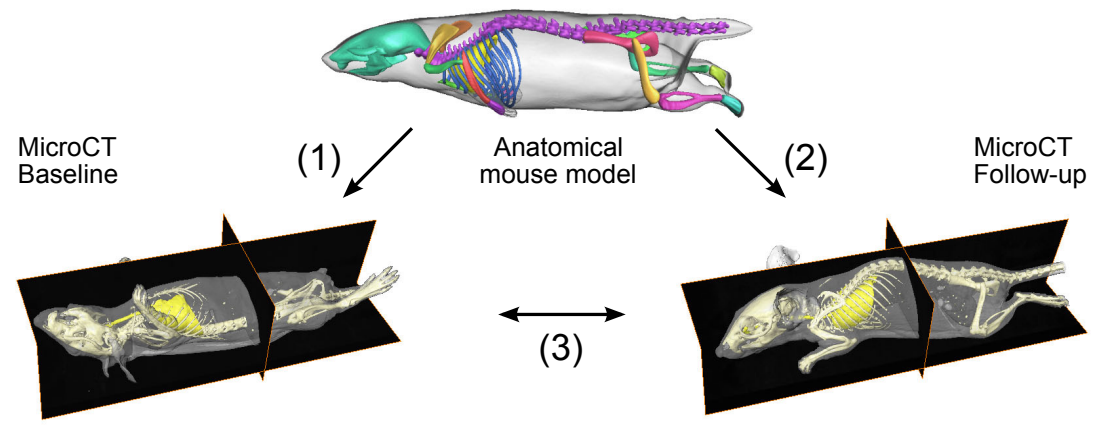

Fig. 2. First, an anatomical animal atlas (skeleton, lungs, skin) is registered to a baseline (fixed image, 1) and one or multiple follow-up (moving image, 2) MicroCT datasets. The point correspondence between the atlas and the datasets allows to establish point correspondences between the datasets as well, which can subsequently be used to regularize intensity-based registrations (3).

\section{Method: Whole-Body Mouse Registration}

In the following, we shortly describe an atlas-based framework for articulated registration presented in earlier work 1] and then the proposed extension for intensity-based registration. An overview of the framework is shown in Fig. 2, The fixed and moving images are denoted with $I_{F}$ and $I_{M}$ respectively, and the transformation relating the two by $\boldsymbol{T}_{\boldsymbol{\mu}}$, with parameters $\boldsymbol{\mu}$.

\subsection{Articulated Whole-Body Registration}

The mouse atlas used in this work is the publicly available MOBY atlas [2] that we modified by manually segmenting individual bones and organs, identifying joint locations and adding anatomically realistic joint models. The registration of this atlas to MicroCT was presented in previous work [1] and will be described briefly. Using a hierarchical anatomical model of the skeleton, each atlas bone is registered individually to an unlabeled skeleton surface representation, using the Iterative Closest Point (ICP) algorithm [8]. In each step, the Degrees of Freedom (DoFs) of the transformation function are defined by the joint type, by which the current bone is connected to the bone that is higher in the hierarchy. To account for differences in bone size, anisotropic scaling is added to the motion parameters of each bone. Thus, the DoFs vary between seven for a hinge joint (translation, non-isotropic scaling, one rotation) and nine for a ball joint. The surfaces of the lungs and the skin are subsequently registered, initialized by the skeleton registration result. The final result is a dense set of corresponding points on the skin, the skeleton and the lungs. Establishing such a point correspondence between the atlas and a target for data of several timepoints, allows to subsequently establish point correspondence between the timepoints as well (see Fig. 2). Corresponding pointsets of two different timepoints are in the following denoted as $\mathcal{Z}_{F}$ and $\mathcal{Z}_{M}$. 


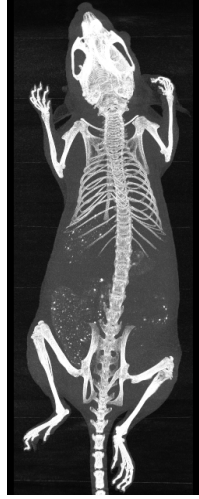

(a) Moving $I_{M}$

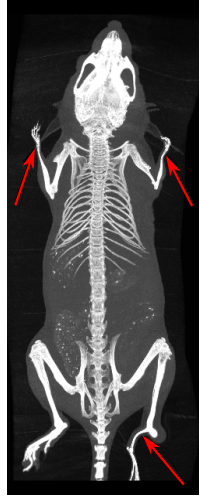

(b) IFFD

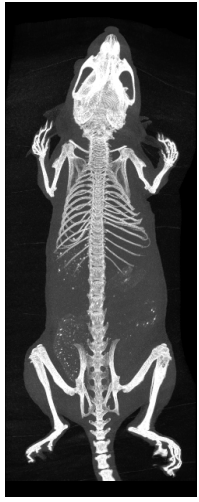

(c) TPS

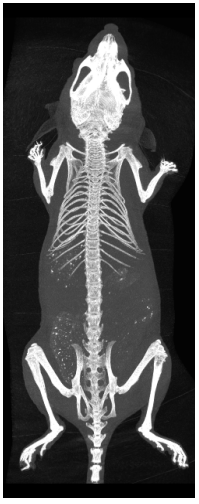

(d) IFFD Reg

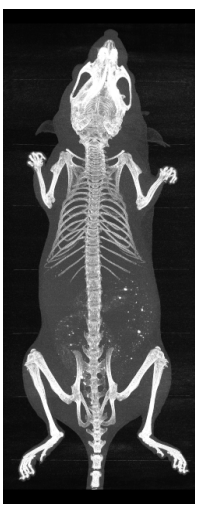

(e) Fixed $I_{F}$

Fig. 3. Dorsal-ventral maximum intensity projections of MicroCT volumes before and after registration with different methods. Note that (a) was acquired in prone, whereas (e) was acquired in supine position. The arrows indicate erroneous limbs after registration based on intensity information only. This is the animal shown in Fig. 5

\subsection{Regularized Intensity-Based Registration}

The articulated skeleton registration is surface-based and mostly neglects intensity information in the data. To combine the robustness of the articulated registration with the accuracy of intensity-based methods, we propose to regularize an intensity-based registration with the point correspondence from the articulated registration. Registration is formulated as an optimization problem:

$$
\arg \min _{\boldsymbol{\mu}} \mathcal{C}=\arg \min _{\boldsymbol{\mu}} \mathcal{S}_{\mathrm{sim}}\left(\boldsymbol{T}_{\boldsymbol{\mu}} ; I_{F}, I_{M}\right)+\alpha \mathcal{S}_{\mathrm{CP}}\left(\boldsymbol{T}_{\boldsymbol{\mu}} ; \mathcal{Z}_{F}, \mathcal{Z}_{M}\right)
$$

where the cost function $\mathcal{C}$ is optimized with respect to the transformation parameters $\boldsymbol{\mu}$. $\mathcal{S}_{\text {sim }}$ measures the image intensity similarity. We chose Normalized Cross Correlation (NCC), because all datasets are acquired with the same modality. We thus assume a linear relationship between the intensity values of $I_{F}$ and $I_{M}$. $\mathcal{S}_{\mathrm{CP}}$ is a metric incorporating the similarity of the corresponding pointsets $\mathcal{Z}_{F}$ and $\mathcal{Z}_{M}$ and is defined as the mean Euclidean distance between them:

$$
\mathcal{S}_{\mathrm{CP}}=\frac{1}{P} \sum_{\boldsymbol{x}_{F}^{i} \in \mathcal{Z}_{F}}\left\|\boldsymbol{x}_{M}^{i}-\boldsymbol{T}_{\boldsymbol{\mu}}\left(\boldsymbol{x}_{F}^{i}\right)\right\|,
$$

where $P$ is the number of corresponding points, and $\boldsymbol{x}_{F}^{i}, \boldsymbol{x}_{M}^{i}$ corresponding points from the fixed and moving image pointsets, respectively. The two terms of Eq. 1 are weighted by the parameter $\alpha$. The optimization problem is solved using a parameter-free Adaptive Stochastic Gradient Descent (ASGD) optimization routine 9], in a multiresolution fashion, using Gaussian pyramids. For each resolution, the optimal value of $\alpha$ is set manually, depending on how much the 

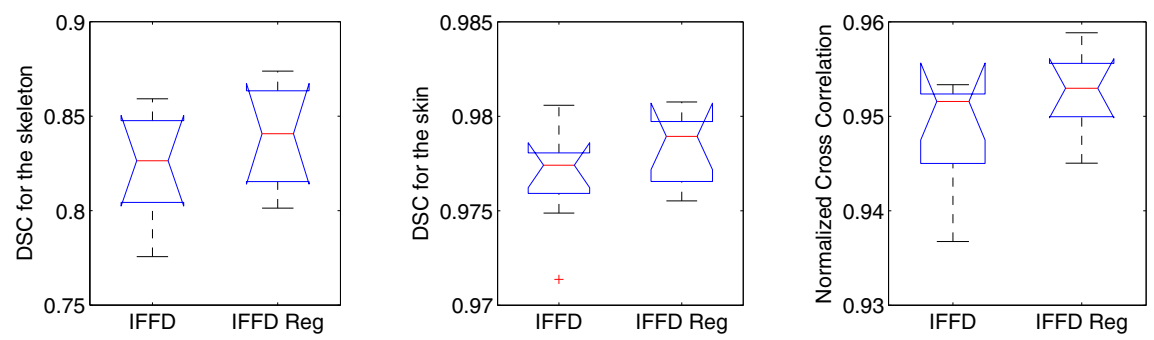

Fig. 4. Boxplots of the DSC for the skeleton and the skin and the NCC, for IFFD and IFFD Reg. Notch overlap indicates no significant difference $(\mathrm{p} \geq 0.05)$ between medians. Note that after initialization, the medians are: DSC skeleton 0.15 , DSC skin 0.81 , NCC 0.65 and using TPS interpolation $0.42,0.91$ and 0.81 .

image intensity and the point distance measure should contribute to $\mathcal{C}$. In the first resolutions, $\mathcal{S}_{\mathrm{CP}}$ should have a relatively large impact on $\mathcal{C}$, to remove large postural differences. Thus, $\alpha$ is set to a relatively large value because otherwise the optimization may get stuck in local minima. Assuming that afterwards $I_{F}$ and $I_{M}$ are coarsely aligned, the influence of $\mathcal{S}_{\mathrm{CP}}$ can be gradually decreased and removed from $\mathcal{C}$ in the last resolution $(\alpha=0)$.

The intensity-based registration was initialized by a similarity registration (motion and isotropic scaling), followed by nonrigid registration with the transformation $\boldsymbol{T}_{\boldsymbol{\mu}}$ parameterized by B-splines [10. They were employed in a multigrid setting, gradually refining the B-spline control point grid over the resolutions.

\section{Experimental Setup}

Eight female mice (Balb/c nu/nu, Charles River, L'Arbresle, France), 6 weeks old at baseline, were scanned twice, three weeks apart, once in prone and once in supine position and with arbitrary limb position. MicroCT (SkyScan 1076, Kontich, Belgium) parameters were: $1.5^{\circ}$ steps, $180^{\circ}, 50 \mathrm{keV}$ x-ray voltage, $200 \mu \mathrm{A}$ anode current, $\mathrm{Al}$ filter $0.5 \mathrm{~mm}$ and exposure time $100 \mathrm{~ms}$. The datasets were reconstructed with built-in software (beam-hardening and ring artifact correction both 10) and a dynamic range of -1000 to 4000 Hounsfield units. No cardiac nor respiratory gating was used. The data was subsampled to $144^{3} \mu \mathrm{m}^{3}$ voxelsize $(\approx 250 \times 200 \times 650$ voxels $)$, smoothed with a Gaussian filter $(\sigma=1)$ and segmented using the Color Structure Code technique 13] with $T=24$ for the skeleton and the skin and $T=6$ for the lungs. Triangular surface meshes were extracted from the segmentations using Marching Cubes (more details in [1]).

Following the procedure in Section [3.1, we derived $\approx 2000$ correspondences on the skeleton, the lungs and the skin. For the intensity-based registration, we used 5 resolutions (500 iterations) for the similarity registration and 6 resolutions (2000 iterations) for the B-Spline registration. $\alpha$ was kept constant at 0.05 in resolutions 1-4, decreased to 0.005 and 0 in resolutions 5 and 6 respectively (parameter files available at http://elastix.isi.uu.nl/wiki.php). Invertibility 
Table 1. Skeleton and skin surface distance and landmark localization accuracy (in voxels). Surface distances are based on eight animals and the landmark distances on a subset of three animals. (*) Results are based on a different, yet comparable dataset.

\begin{tabular}{|l||l|l|l|l|}
\hline Skeleton distance & Mean & Median & Max & Min \\
\hline Init & $9.70 \pm 11.68$ & 5.59 & 81.16 & $3 \mathrm{e}-6$ \\
\hline TPS & $2.01 \pm 2.72$ & 1.32 & 36.91 & $3 \mathrm{e}-6$ \\
\hline IFFD & $1.19 \pm 5.15$ & 0.34 & 71.99 & $5 \mathrm{e}-7$ \\
\hline IFFD Reg & $0.49 \pm 0.80$ & 0.33 & 17.83 & $3 \mathrm{e}-7$ \\
\hline Li et. al 5 $\left(^{*}\right)$ & $0.61 \pm 0.19$ & N/A & N/A & N/A \\
\hline Skin distance & Mean & Median & Max & Min \\
\hline Init & $9.56 \pm 10.30$ & 6.46 & 76.62 & $9 \mathrm{e}-6$ \\
\hline TPS & $3.79 \pm 3.63$ & 2.71 & 36.70 & $1 \mathrm{e}-6$ \\
\hline IFFD & $1.37 \pm 4.58$ & 0.50 & 68.64 & $4 \mathrm{e}-7$ \\
\hline IFFD Reg & $0.83 \pm 1.16$ & 0.49 & 16.41 & $9 \mathrm{e}-8$ \\
\hline \hline Landmark distance & Mean & Median & Max & Min \\
\hline Init & $65.24 \pm 32.81$ & 64.52 & 131.62 & 4.91 \\
\hline TPS & $6.25 \pm 3.75$ & 5.52 & 25.63 & 2.17 \\
\hline IFFD & $3.75 \pm 7.46$ & 1.90 & 51.87 & 0.37 \\
\hline IFFD Reg & $1.97 \pm 1.72$ & 1.57 & 11.51 & 0.37 \\
\hline Li et. al $\mathbf{1 1}(*)$ & $3.46 \pm 1.88$ & 3.64 & 5.96 & 1.04 \\
\hline
\end{tabular}

and smoothness of all final transformations was confirmed using the determinant of the Jacobian of the deformation fields, which was $>0$ within all animals.

For evaluation, the following metrics were chosen: Normalized Cross Correlation (NCC) to assess the intensity similarity and the Dice Similarity Coefficient (DSC) to assess skeleton and skin segmentation accuracy. The DSC is defined as $2\left(V_{1} \cap V_{2}\right) /\left(V_{1}+V_{2}\right)$ and measures structural overlap. It is well suited for elongated and thin structures, which occur in our data (Fig. 1). We also determined the Euclidean Point to Surface Distance (EPSD) between the skeletons and skins of registered datasets. We excluded the tail, since it is irrelevant for most studies. Color-coded EPSD mapping to the surfaces allows to detect local registration inaccuracies. Finally, we assessed how well specific bone structures are registered, by measuring the Euclidean Point to Point Distance (EPPD) between 19 anatomical landmarks, manually indicated before and after registration, on distal body parts like the limbs, on the spine and on the ribs. Results are given after initialization, TPS interpolation, intensity-based registration without (IFFD) and with using regularization (IFFD Reg). For comparison with published work, we present results of $\mathrm{Li}$ et al. [5], because their datasets are comparable to ours.

Correspondence determination was done with Matlab 2010b (The Mathworks, Natick, USA) and the intensity-based registration using the ITK-based and publicly available elastix software [12] on an Intel Xeon E5620 8 cores $(2.4 \mathrm{GHz})$ and $24 \mathrm{~GB}$ RAM. The time requirements were $\approx 5$ mins. for IFFD and $\approx 17$ mins. for IFFD Reg. (including $\approx 5$ mins. to determine correspondence).

\section{Results and Discussion}

Qualitative results of the registration are shown in Fig. 3, quantitative results for the DSC and the NCC are presented in Fig. 4 and the surface distances and 
landmark localization accuracy before and after registration are given in Tab. 1 and Fig. 5. The very large difference between the metrics after initialization and after IFFG are an indication for the large postural differences between the animals. Comparing TPS and IFFD, the average error is smaller for IFFD, but the maximum is much larger. The reason is the large initial postural differences between animals. TPS can deal with that and therefore, all body parts are registered equally well. IFFD is very accurate, when body parts lie within the registration capture range, but fails completely otherwise. Generally, the more distal to the body, the higher the error becomes. Fig. 5 and Fig. 3 support this because the error increases significantly at the limbs. The results of IFFD Reg reveal that our approach can handle large variability in the data without losing accuracy. The DSC plot (Fig. 4) shows excellent overlap for the skeleton and the skin. We obtain subvoxel accuracy for bone and the skin in the surface distance measure (Tab. 1). The maximum distances mainly stem from the very distal ends of the limbs and the ribs for the skeleton, and folds for the skin (Fig. 5). In addition, IFFD Reg yields higher intensity similarity than IFFD (Fig. 4). For all presented metrics, IFFD Reg outperforms both, TPS and IFFD, proving that relying on point correspondence or intensity only is not sufficient for highly accurate registration, in case of large postural differences.

Compared to published data by Li et al. [5]11, we have similar results for the skeleton distance and better results for the landmark localization. Their method pays special attention to registration of the ribs, thus it might yield more accurate results for these structures. However, they evaluate using ex vivo data, excluding rib movement artifacts. If accurate rib registration is required, an additional stiffness penalty could easily be added to our registration criterion 3]. In addition, we want to stress that the method in $\mathrm{Li}$ et al. requires 260 minutes for registration and our method takes $\approx 17$ minutes. We realize that those experiments were performed on outdated hardware (Pentium PC, 2GHz, 1GB RAM), but most of our code was executed single-threaded and in addition, our image domain was approximately twice as big. It would be interesting to compare our method to the promising approach of Suh et al. [6] as well, which seems to be more time efficient and more accurate, compared to Li et al.
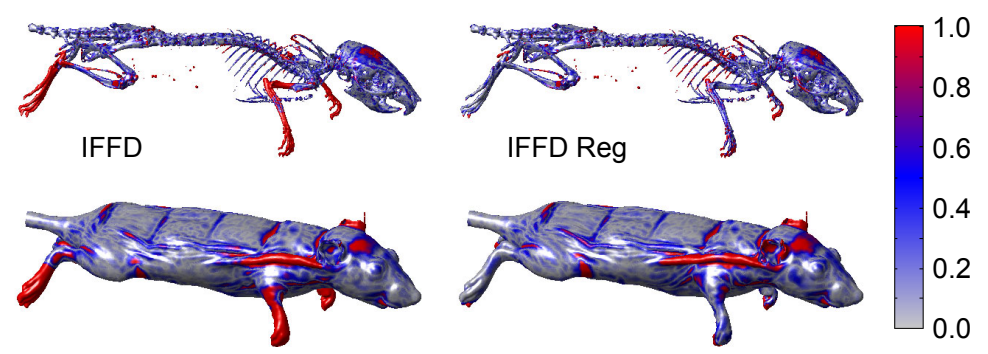

Fig. 5. The skeleton and the skin of an animal at baseline with color-coded Euclidean distance to the nearest surface point on the mapped skeleton and skin after registration using IFFD and IFFD Reg respectively. Values (in voxels) are based on one animal. 
Finally we want to point out, that the registration of an atlas yields a segmentation of the skeleton as a by-product.

\section{Conclusion}

We presented a highly robust and accurate approach for registration of articulated objects with application to whole-body MicroCT data of mice. This we obtained by regularizing an intensity-based registration criterion with a distance metric, derived from point correspondence among datasets. We performed registration of in vivo whole-body MicroCT data with high resolution in $\approx 17$ minutes and obtained subvoxel accuracy for the skeleton and the skin. Compared to competing methods, our approach is very time efficient.

\section{References}

1. Baiker, M., Milles, J., Dijkstra, J., Henning, T.D., Weber, A.W., Que, I., Kaijzel, E.L., Löwik, C.W.G.M., Reiber, J.H.C., Lelieveldt, B.P.F.: Atlas-based whole-body segmentation of mice from low-contrast Micro-CT data. Med. Image Anal. 14(6), 723-737 (2010)

2. Segars, W.P., Tsui, B.M.W., Frey, E.C., Johnson, G.A., Berr, S.S.: Development of a $4 \mathrm{D}$ digital mouse phantom for molecular imaging research. Mol. Im. Biol. 6(3), 149-159 (2004)

3. Staring, M., Klein, S., Pluim, J.P.W.: A Rigidity Penalty Term for Nonrigid Registration. Med. Ph. 34(11), 4098-4108 (2007)

4. Somayajula, S., Joshi, A.A., Leahy, R.M.: Mutual Information Based Non-rigid Mouse Registration Using A Scale-Space Approach. In: Proc. IEEE Int. Symp. on Biomedical Imaging, pp. 1147-1150. IEEE Press, New York (2008)

5. Li, X., Yankeelov, T.E., Peterson, T.E., Gore, J.C., Dawant, B.M.: Automatic nonrigid registration of wholebody CT mice images. Med. Ph. 35(4), 1507-1520 (2008)

6. Suh, J.W., Scheinost, D., Dione, D.P., Dobrucki, L.W., Sinusas, A.J., Papademetris, X.: A non-rigid registration method for serial lower extremity hybrid SPECT/CT imaging. Med. Image Anal. 15(1), 96-111 (2011)

7. Papademetris, X., Dione, D.P., Dobrucki, L.W., Staib, L.H., Sinusas, A.J.: Articulated rigid registration for serial lower-limb mouse imaging. In: Duncan, J.S., Gerig, G. (eds.) MICCAI 2005. LNCS, vol. 3750, pp. 919-926. Springer, Heidelberg (2005)

8. Besl, P.J., McKay, N.D.: A method for registration of 3D shapes. IEEE Trans. Pattern Anal. Machine Intelligence 14(2), 239-256 (1992)

9. Klein, S., Pluim, J.P.W., Staring, M., Viergever, M.A.: Adaptive stochastic gradient descent optimisation for image registration. J. Com. Vis. 81(3), 227-239 (2009)

10. Rueckert, D., Sonoda, L.I., Hayes, C., Hill, D.L.G., Leach, M.O., Hawkes, D.J.: Nonrigid registration using free-form deformations: application to breast MR images. IEEE TMI 18(8), 712-721 (1999)

11. Li, X., Yankeelov, T.E., Peterson, T.E., Gore, J.C., Dawant, B.M.: Constrained non-rigid registration for whole body image registration: method and validation. In: Cleary, K.R., Miga, M.I. (eds.) SPIE Med. Im., vol. 6512, pp. 11-18 (2007)

12. Klein, S., Staring, M., Murphy, K., Viergever, M.A., Pluim, J.: Elastix: a toolbox for intensity-based medical image registration. IEEE TMI 29(1), 196-205 (2010)

13. Sturm, P., Priese, L., Wang, H.: A CSC Based Classification Method for CT Bone Images. In: Proc. 3DPVT, pp. 1080-1084. IEEE Press, New York (2006) 